\title{
Initial observations of auroras by the multi-spectral auroral camera on board the Reimei satellite
}

\author{
Yasuyuki Obuchi ${ }^{1,5}$, Takeshi Sakanoi ${ }^{1}$, Atsushi Yamazaki ${ }^{2}$, Tomohiro Ino ${ }^{1,6}$, Shoichi Okano ${ }^{1}$, Yasumasa Kasaba ${ }^{3}$, \\ Masafumi Hirahara ${ }^{4}$, Yoshikazu Kanai ${ }^{5}$, and Norihide Takeyama ${ }^{5}$ \\ ${ }^{1}$ Planetary Plasma and Atmospheric Research Center, Graduate School of Science, Tohoku University, Japan \\ ${ }^{2}$ Institute of Space and Astronautical Science, Japan Aerospace Exploration Agency, Kanagawa, Japan \\ ${ }^{3}$ Department of Geophysics, Graduate School of Science, Tohoku University, Japan \\ ${ }^{4}$ Department of Earth and Planetary Science, Graduate School of Science, The University of Tokyo, Japan \\ ${ }^{5}$ Genesia Corporation, Japan \\ ${ }^{6}$ Canon Corporation, Japan
}

(Received January 11, 2008; Revised June 21, 2008; Accepted June 21, 2008; Online published September 8, 2008)

\begin{abstract}
The small Japanese scientific satellite Reimei was launched successfully from Baikonur Space Center into a sun-synchronous (noon-midnight meridian) polar orbit at an altitude of $\sim 630 \mathrm{~km}$ on August 23, 2005. Auroral fine structure observations have been performed by the multi-spectral auroral camera (MAC) and electron/ion spectrum analyzers (ESA/ISA) on board Reimei. The MAC is a three channel camera system used to simultaneously observe the temporal dynamics of the auroral fine structure at the wavelengths of $428 \mathrm{~nm}\left(\mathrm{~N}_{2}^{+} 1 \mathrm{NG}\right)$, $558 \mathrm{~nm}(\mathrm{O})$, and $670 \mathrm{~nm}\left(\mathrm{~N}_{2}\right.$ 1PG). Since its successful launch, Reimei has been operated continuously and produced lots of unique data on the auroral fine structures with the MAC and ESA/ISA. The initial observation data from MAC has shown the temporal dynamics of auroral fine structure, such as the vortex street, appearing in the poleward edge of the auroral oval. The significant differences in the auroral shapes between the MAC channels suggest the existence of different emission processes. Further, the height profiles of the aurora and airglow are clearly seen in the image data in the limb direction. It is expected that the various mechanisms and dynamics of auroral fine structures can be investigated by analyzing the Reimei observation data.
\end{abstract}

Key words: Auroral fine structure, visible camera.

\section{Introduction}

It is well known that auroral dynamics are strongly coupled with magnetospheric and ionospheric phenomena, and these have been investigated with immense interest by using ground-based, satellite, and rocket observation data. In particular, the field-aligned acceleration processes and magnetosphere-ionospheric coupling have been examined with particle, magnetic, and electric fields, and with plasma wave data obtained by various satellites, such as DE, S33, AKEBONO, Freja, and FAST (Hoffman and Schmerling, 1981; Cattell, 1982; Oya and Tsuruda, 1990; Lundin et al., 1994; Sakanoi et al., 1995; Carlson et al., 1998). The auroral electrons precipitating from the magnetosphere are accelerated by the field-aligned quasi-electrostatic electric field (convergent electric field), called the inverted-V structure, and also by inertial Alfven waves (Schriver et al., 2003) at altitudes above a few thousands $\mathrm{km}$. As reported by Frey et al. (1998), it is suggested that a discrete aurora appears as a result of electron precipitation accelerated by the convergent electric field.

Previous ground-based measurements have shown that an optical aurora has a fine structure down to a $100 \mathrm{~m}$ width (Maggs and Davis, 1968; Borovsky, 1993). On the other

Copyright (c) The Society of Geomagnetism and Earth, Planetary and Space Sciences (SGEPSS); The Seismological Society of Japan; The Volcanological Society of Japan; The Geodetic Society of Japan; The Japanese Society for Planetary Sciences; TERRAPUB hand, particle data taken by satellites have shown that the typical width of an inverted-V structure is several tens to a hundred km (Sakanoi et al., 1995), and rocket experiments have shown the existence of a small-scale inverted- $\mathrm{V}$ with a width of a few $\mathrm{km}$ (McFadden et al., 1999). Therefore, there is a significant discrepancy between the optical aurora obtained from ground-based observation data and the precipitating electrons obtained by satellite and rocket measurement data. The fundamental reason for this discrepancy is that it is extremely difficult to make simultaneous measurements of an optical aurora and its precipitating electrons with high-temporal and high-spatial resolutions. In the case of ground-based imaging data, the spatial resolution is degraded due to overlapping auroral structures along a line-of-sight direction. It is also difficult to have an opportunity for conjugate measurements between an optical aurora and its precipitating electrons along a magnetic field line connecting satellite and ground-based experiments.

The ultimate solution to this problem is simultaneous imaging and particle measurements of an aurora with hightemporal and spatial resolutions by a single satellite. The optical imager and particle instruments on board the Reimei satellite enable us to make such simultaneous observations with high temporal and spatial resolutions by pointing the field of view of the MAC at the magnetic footprint threading the satellite. Sakanoi et al. (2003) described the performance of the MAC in the mid-course phase of its devel- 


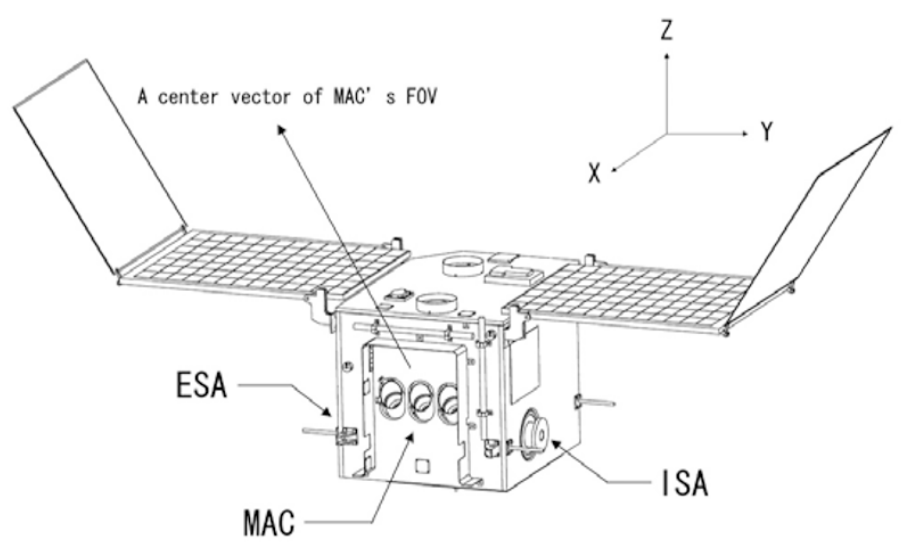

Fig. 1. Schematic view of the Reimei satellite and locations of the MAC and ESA/ISA. A center vector of the FOV of the MAC is at a 45 degree slant in the $Z-X$ plane.

opment, while the definitive performance of the MAC and initial observation data are given in this paper.

\section{Instrumentation}

\subsection{Reimei satellite}

The Reimei satellite is the first small scientific satellite developed by JAXA/ISAS. Its size and weight are $724 \mathrm{~mm}$ $\times 626 \mathrm{~mm} \times 609 \mathrm{~mm}$ and about $70 \mathrm{~kg}$, respectively. The scientific purpose of Reimei is to investigate the auroral fine structure. Reimei was launched from Baikonur Space Center into a sun-synchronous polar orbit with an inclination of 98.6 degree and an altitude of approximately $630 \mathrm{~km}$ on August 23, 2005. The orbital meridian is on 0050-1250 local time (Saito et al., 2005), and the orbital period is about 98 minutes. A three-channel monochromatic auroral camera (MAC) (Sakanoi et al., 2003) and top-hat type electron and ion energy spectrum analyzers (ESA/ISA) (Asamura et al., 2003) are on board.

The simultaneous observation data for an aurora's image and particles are obtained by these two instruments with high-temporal and spatial resolutions. In addition, three Langmuir probes, which are called the plasma current monitor (CRM), and a magnetic field sensor (GAS) are also on board. GAS is used for deriving the attitude vector of the satellite. An external view of Reimei with the MAC and ESA/ISA configuration is shown in Fig. 1. The attitude control of the satellite is accomplished by a 3-axis bias momentum control using three magnetic torquers and a reaction wheel. This allows Reimei to point in any desired orientation with high accuracy. The only limit on the attitude control comes from the need for the solar panel to face the sun within \pm 25 degrees in order to obtain sufficient power. For attitude determination, several sensors are installed: a star tracker, a spin sun sensor, a non-spin sun sensor, an optical fiber gyro, and a magnetic field sensor. The attitude measurement data from these sensors allows an attitude determination with an accuracy better than $\sim 0.05$ degrees. This accuracy represents a width of about $460 \mathrm{~m}$ at an auroral height. Accordingly, the MAC can aim at the footprint of the magnetic field line threading the satellite with an accuracy of about $1 \mathrm{~km}$, and the imaging and particle observation data for an auroral fine structure can be obtained simultaneously. In addition, the MAC also has a capability to observe the altitude distribution of an aurora emission by aiming its field of view at the earth's limb.

\subsection{Development of MAC}

The MAC is a three-channel monochromatic CCD imager. A picture of a MAC flight model is shown in Fig. 2, and a schematic drawing of its cross-section is shown in Fig. 3. The specifications for the MAC are summarized in Table 1. Because Reimei is a small satellite, the MAC had to be made compact, with a light weight. Due to the weight limitation, the camera body consists of six thin aluminum plates with rib structures that can tolerate the launch conditions. These plates are finished by black chemglaze in order to reduce stray light scattering. Since the MAC has three independent optical systems (refracting optics), it can observe three auroral emissions simultaneously. The selected emission lines are $\mathrm{N}_{+}^{2} 1 \mathrm{NG}(427.8 \mathrm{~nm}), \mathrm{O}$ green line $(557.7 \mathrm{~nm})$, and $\mathrm{N}_{2} 1 \mathrm{PG}(670 \mathrm{~nm})$. In order to observe auroral fine structures with high-temporal and spatial resolutions, $427.8 \mathrm{~nm}$ and $670 \mathrm{~nm}$ emissions (permitted lines), whose lifetimes are very short, were selected. The forbidden $557.7 \mathrm{~nm}$ emission was chosen because its auroral excitation process is known to be related to $\mathrm{N}_{2} 1 \mathrm{PG}$ emission. In order to investigate the auroral fine structures properly, it is necessary to observe these emissions with a short exposure cycle by using bright

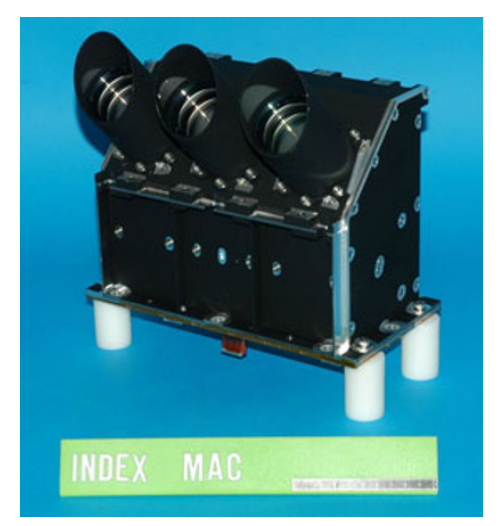

Fig. 2. A picture of the MAC flight model. 
Table 1. Specifications for the MAC.

\begin{tabular}{|c|c|c|}
\hline Item & & Specification \\
\hline Type & & ing monochromatic imager \\
\hline Channel & $3(3$ sets & tics, band-pass filters, and CCDs) \\
\hline Wavelength & $427.8 \mathrm{~nm}$ & $\mathrm{~N}), 557.7 \mathrm{~nm}(\mathrm{OI}), 670 \mathrm{~nm}\left(\mathrm{~N}_{2} 1 \mathrm{P}\right)$ \\
\hline Focal length & & $50 \mathrm{~mm}$ \\
\hline$F$ number & & 1.5 \\
\hline FOV & & $7.6^{\circ}$ \\
\hline Observation mode & \multicolumn{2}{|c|}{4 modes (see details in Table 3 ) } \\
\hline \multirow{3}{*}{$\mathrm{CCD}$} & Type & Interline transfer and surface irradiation type \\
\hline & Pixel number & $1024 \times 1024$ pixels \\
\hline & Quantum efficiency & $\sim 0.6 @ 558 \mathrm{~nm}$ \\
\hline Mass & \multicolumn{2}{|r|}{$4585 \mathrm{~g}$} \\
\hline Electrical power & \multicolumn{2}{|r|}{$20.4 \mathrm{~W}$} \\
\hline
\end{tabular}

Table 2. FWHM and transmittance of band-pass filters.

\begin{tabular}{c|cc}
\hline Center wavelength & FWHM & Transmittance (center of bandwidth) \\
\hline $427.8 \mathrm{~nm}$ & $2.5 \mathrm{~nm}$ & 0.46 \\
$557.7 \mathrm{~nm}$ & $1.57 \mathrm{~nm}$ & 0.55 \\
$670.0 \mathrm{~nm}$ & $38.23 \mathrm{~nm}$ & 0.90 \\
\hline Lens & - & 0.8 \\
\hline \multicolumn{2}{c}{ The transmittance of the total optical assembly is computed by multiplying } \\
the transmittance of each filter by the lens.
\end{tabular}

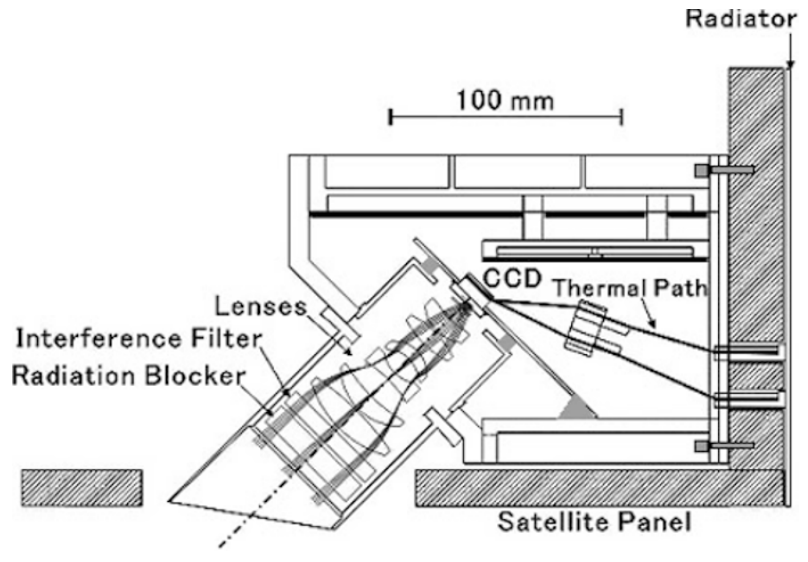

Fig. 3. Schematic drawing of a cross section of the MAC and its surroundings. Three CCDs are connected to the radiator, which is attached to the outside of the satellite panel, with thermal paths.

optics with little aberration. Each optical assembly of the MAC consists of six synthetic silica lenses, an interference filter, and a radiation shield. The radiation shield is a flat plate made of synthetic silica. These optics were designed and fabricated by the Genesia corporation, and their aberrations were minimized. The transmittance of the whole optical system, excluding the interference filter, is 0.8 . The optical focal length is $50 \mathrm{~mm}$, and the $F$ number is 1.5 . The full field of view of each channel is made as small as 7.6 degrees in order to observe fine scale auroral structures. When the MAC observes aurora emissions by pointing its field of view at the magnetic footprint, the mapping area is about $70 \mathrm{~km}$ square, assuming that an aurora height is $110 \mathrm{~km}$. The interference filters were made by the Andover corporation. The FWHM and transmittance of each filter are given in Table 2. Each optical element is mounted in a cylindrical barrel, which is made of aluminum (A6061T) and black anodized in order to reduce any stray light. Furthermore, an aluminum hood finished by black chemglaze was put on each optical barrel. The CCD chip alignment on the focal plane, under vacuum conditions, was precisely adjusted by a collimator and gimbals. The alignment error in the entire CCD area should be within $10 \mu \mathrm{m}$ of the focal plane. The tilt angle of the sensor against the optical axis was obtained from the focused images of point light sources at each corner of the CCD using the equipment. Then, the tilt angle could be compensated for by correcting the size of the shims inserted into the bushings that support the CCD electronic board. In addition, after vibration and shock tests that simulated launch conditions, a focus check was carried out again to see whether the correct alignment of the CCD surface was maintained. Finally, it was verified that the optical alignment and focal position did not change through these environmental tests.

A diagram of the instrument control and data flows for the satellite is shown schematically in Fig. 4. The camera electronics of the MAC consist of three CCD boards with two control boards. These five boards are connected together by flexible interface lines. One of the two control boards is used for power control and the one is for digital data control. The power control board supplies the power, which is fed from the Science-Handling-Unit (SHU) on Reimei, to each CCD board and the digital data control board. The latter board handles digital signals between the CCD and SHU, and controls the exposure time and data transfer. The exposure time can be selected logarithmically between $130 \mu \mathrm{sec}$ and $3 \mathrm{sec}$ by sending commands from the ground. In a typical observation looking down at a magnetic footprint, the exposure time is $60 \mathrm{msec}$ considering 
Table 3. Specifications for each observation mode.

\begin{tabular}{|c|c|c|c|c|}
\hline Mode & Aim & Pixel binning & Temporal resolution & Spatial resolution \\
\hline Mode-0 & Footprint & $16 \times 16$ pixels & msec & $1.2 \times 1.2 \mathrm{~km}$ \\
\hline Mode-1 & Limb of the earth & $16 \times 16$ pixels & $\sec$ & $2.4 \times 2.4 \mathrm{~km}$ \\
\hline Mode-2 & Stars & $2 \times 2$ pixels & $4.04 \mathrm{sec}$ & - \\
\hline Mode-3 & Limb of the earth & $8 \times 8$ pixels & msec & $1.2 \times 1.2 \mathrm{~km}$ \\
\hline
\end{tabular}

Mode-2 is used for alignment calibration.

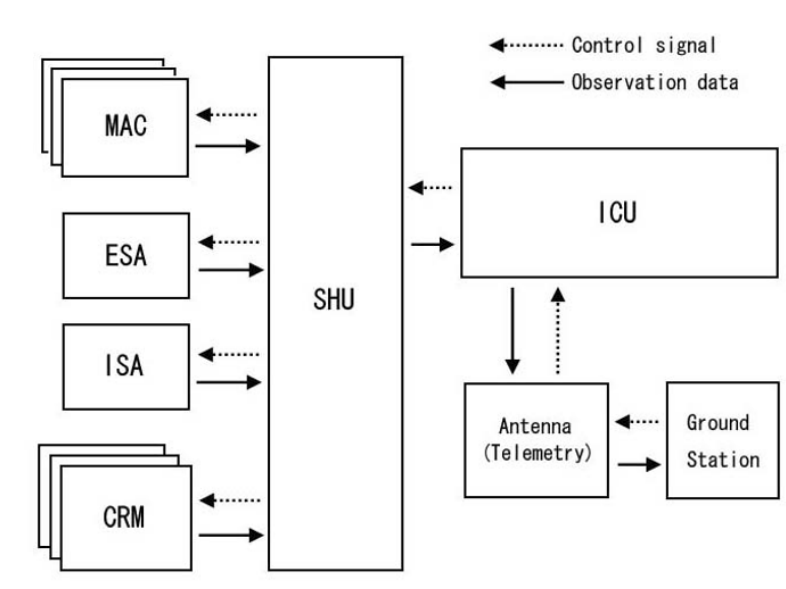

Fig. 4. Schematic diagram of the control and data flows of the satellite instruments and central satellite system. Dashed and solid lines represent the control signal and observation data flows, respectively.

the orbital velocity of Reimei, which is about $8 \mathrm{~km} / \mathrm{sec}$. The $\mathrm{CCD}$ is an interline transfer and front illuminated type with $1024 \times 1024$ pixels (pixel size is $6.4 \mu \mathrm{m} \times 6.4 \mu \mathrm{m}$ ), and three kinds of pixel binning can be used, which give different spatial resolutions (see Section 2.3 and Table 3). The quantum efficiency of the CCD is about 0.6 at $557.7 \mathrm{~nm}$. Because the temperature of the CCD and filter affects the observation performance, it is necessary to monitor their temperatures accurately. In order to monitor the temperatures from the ground, seven temperature sensors are used, attached to the barrel of each lens, each CCD board, and the power control board. These temperature sensors are driven by the two above mentioned control boards, and the temperature data are sent to the SHU, along with the status data for the MAC. The temperature data are integrated into observation data every 30 seconds by the SHU, and transferred to the ground by telemetry. The observation data handling and power for all of the scientific instruments on board Reimei are controlled by the SHU. The memory size of the SHU for storing observation data is 64 Mbytes, which limits the simultaneous observation duration time of the MAC and ESA/ISA to 7 minutes. This time limit corresponds to 26 degrees of latitude. Since the downlink speed is low and the downlink time is short, the observation data of the MAC are compressed by the SHU, which converts it from 12-bit linear data to 8-bit logarithmic data. The SHU compresses the 12 bit MAC data into an 8 bit depth logarithmically. In addition, the size of the science data is also compressed in the Integrated Control Unit (ICU), which controls all of the instruments on board Reimei, before it is sent to the ground by telemetry.

Because the camera electronics of the MAC are made from off-the-shelf commercial products, their performance in a space environment was not guaranteed and it was necessary to confirm their performance in a simulated space environment and to make adjustments if something was wrong. At the first stage of this confirmation, it was seen from infrared images of the electronics boards in operation, that the temperatures of some elements on the CCD and power control boards were over 60 degrees Celsius. Because these temperatures were close to the upper limit of the operation temperature range, it was supposed that these elements would become hotter than the limit in space. In order to cool the high temperature elements, thermal paths were introduced, which are made of aluminum and copper mesh sheets. In addition, indium foil was inserted between the high temperature elements and these thermal paths in order to improve the heat transfer efficiency. After this treatment, an operation test in vacuum conditions was performed for 11 minutes and it was confirmed that the temperatures were suppressed to values lower than the limit. Second, the CCD needs to be cooled to less than 0 degrees Celsius for a sufficient signal to noise ratio. Because the CCD on the MAC does not have a thermoelectric cooling device, a thermal path was attached to the CCD. This is composed of 20 stacked graphite sheets $(0.1 \mathrm{~mm}$ thickness each) with oxygen-free copper blocks and aluminum parts at the CCD ends. Using the thermal path, the CCD is thermally connected to a radiation-plate, which is made of aluminum coated with silver evaporated Teflon tape. The radiation-plate is placed to face the space right behind the satellite panel and is cooled by radiation cooling. The indium foils are attached to both ends of the thermal path to increase thermal conductivity. The CCD temperature was simulated by using a thermal model. In addition, a thermal vacuum test was performed to check whether the CCD was cooled as expected and whether its dark noise could be reduced sufficiently to observe the weak auroral emission. The thermal vacuum test was also meant to check whether the MAC could show sufficient performance in the space environment. On this test, by changing the temperature inside a thermostatic vacuum chamber from -10 to 20 degrees Celsius, dark noise data were measured. By using these data as a reference, the image data obtained by the MAC in orbit have been processed. As a test result, the thermal paths worked quite well, the CCD could be cooled to lower than 0 degrees Celsius, and therefore, the MAC was expected to work well in the orbital environment. Actually, after the launch of Reimei, it was confirmed that the CCD was cooled to below -7 degrees Celsius. Third, since it 
Table 4. Sensitivity and dynamic range of the MAC.

\begin{tabular}{cccc}
\hline Observation mode & Wavelength & Sensitivity $[\mathrm{R} /$ count/ms $]$ & Dynamic range $[\mathrm{R}]$ \\
\hline \multirow{2}{*}{ Mode-0 } & $427.8 \mathrm{~nm}$ & $8.14 \times 10^{3}$ & $0-321 \times 10^{3}$ \\
& $557.7 \mathrm{~nm}$ & $5.99 \times 10^{3}$ & $0-219 \times 10^{3}$ \\
& $670 \mathrm{~nm}$ & $5.63 \times 10^{3}$ & $0-210 \times 10^{3}$ \\
\hline \multirow{2}{*}{ Mode-1 } & $427.8 \mathrm{~nm}$ & $8.14 \times 10^{3}$ & $0-63.2 \times 10^{3}$ \\
& $557.7 \mathrm{~nm}$ & $5.99 \times 10^{3}$ & $0-42.8 \times 10^{3}$ \\
& $670 \mathrm{~nm}$ & $5.63 \times 10^{3}$ & $0-41.2 \times 10^{3}$ \\
\hline \multirow{2}{*}{ Mode-2 } & $427.8 \mathrm{~nm}$ & $525 \times 10^{3}$ & $0-1.94 \times 10^{9}$ \\
& $557.7 \mathrm{~nm}$ & $394 \times 10^{3}$ & $0-1.33 \times 10^{9}$ \\
& $670 \mathrm{~nm}$ & $363 \times 10^{3}$ & $0-1.25 \times 10^{9}$ \\
\hline \multirow{2}{*}{ Mode-3 } & $427.8 \mathrm{~nm}$ & $32.8 \times 10^{3}$ & $0-172 \times 10^{3}$ \\
& $557.7 \mathrm{~nm}$ & $24.3 \times 10^{3}$ & $0-117 \times 10^{3}$ \\
& $670 \mathrm{~nm}$ & $22.9 \times 10^{3}$ & $0-116 \times 10^{3}$ \\
\hline
\end{tabular}

was thought that both the CCD and interference filter were likely to be damaged by radiation in the space environment, a radiation test was performed with $100 \mathrm{MeV}$ protons. The total dose in this test was $9 \mathrm{krad}$, which is equivalent to the amount expected during three years in orbit. Based on this test, the damage to the CCD and the decrease in filter transmittance caused by the browning of the glass substrate were evaluated and the radiation damage was regarded to be negligible for the observation. In the way described above, the MAC was qualified for use as the onboard imager for a satellite tasked with observing auroral fine structures.

\subsection{Observation mode of MAC}

The MAC has four observation modes. These modes are called "Mode-0," "Mode-1," "Mode-2," and "Mode-3." The temporal and spatial resolutions of each mode are summarized in Table 3.

"Mode- 0 " is the observation mode for the simultaneous observation of an auroral image and particle measurements. In this mode, $16 \times 16$ pixels are binned, making the total number of bins $64 \times 64$. Due to this binning, the spatial resolution for this mode is about $1.2 \mathrm{~km}$, assuming that the FOV of the MAC is directed at the magnetic footprint, and that the auroral altitude is about $110 \mathrm{~km}$. Considering the orbital motion of Reimei, which has a velocity of $8 \mathrm{~km} / \mathrm{s}$, and the spatial resolution mentioned above, the exposure cycle of this mode was chosen to be $120 \mathrm{msec}$. The exposure time can be selected between $130 \mu \mathrm{sec}$ and $106 \mathrm{msec}$, and the typical exposure time is $60 \mathrm{msec}$.

"Mode-1" is selected in a case where the MAC is used to observe the altitude distribution of an aurora emission while pointing the FOV at the earth's limb. In this mode, the pixel binning of the CCD is similar to that of "Mode-0." However, since an aurora would be located far away from Reimei, the spatial and temporal resolutions are different from those of "Mode-0." Thus, the spatial resolution is about $4 \mathrm{~km}$, and the exposure cycle is $1 \mathrm{sec}$. The typical exposure time is $480 \mathrm{msec}$, since the auroral intensity is expected to be weaker than that seen along the magnetic field line with Mode- 0 .

"Mode-2" is used for calibration of the system. The MAC is used to observe stars, ground structures, and coastlines in this mode, in order to determine the absolute direction of pointing. By observing these targets, the angles between the MAC's FOV and the three satellite coordinate axes can be determined. After the launch, we could obtain the exact orientation of each optical axis for each channel by analyzing the image data for stars and land features obtained by the MAC with this observation mode. The pixel binning in this mode is $2 \times 2$ pixels, and the total number of bins is $512 \times 512$. Due to this binning, the spatial resolution is about $150 \mathrm{~m}$ when the MAC points to a sub-satellite point. The exposure cycle is $4 \mathrm{sec}$, since the data size in this mode is much larger than those of "Mode- 0 " and "Mode- 1 " and a longer time is needed for data transfer from the MAC to the SHU. The exposure time is about $1.9 \mathrm{sec}$ when observing a star with a magnitude brighter than 5 .

"Mode-3" is used for the observation of the height distribution of an aurora with higher spatial resolution than that of "Mode-0" or "Mode-1." However, the sensitivity of this mode is lower than that with "Mode-0" or "Mode-1." The pixel binning in this mode is $8 \times 8$ pixels, and the total number of bins is $128 \times 128$. Due to this binning, the spatial resolution of the MAC in this mode is twice that of "Mode0 " and "Mode-1." The exposure cycle is $480 \mathrm{msec}$, which is longer than that of "Mode-0." If the appearance of a bright aurora is predicted, an observation plan with this mode can be prepared.

\subsection{Sensitivity of the MAC}

After the assembly of the MAC and its environmental tests, a sensitivity calibration was performed at the National Institute of Polar Research by using an integrating sphere with a diameter of $2 \mathrm{~m}$. The calibration results are summarized in Table 4. The formula and factors used for the calibration are as follows;

$$
N=I \frac{10^{6}}{4 \pi} A \Omega T \eta t E
$$

where $N$ is the signal count of a single bin in an image data, $I$ is the emission intensity (Rayleigh), $A$ is the effective area of the MAC's optics $\left(8.72 \mathrm{~cm}^{2}\right), \Omega$ is the solid angle extended to a single bin from the objective lens (str), $T$ is the transmittance of the optics, $\eta$ is the quantum efficiency of the CCD, $t$ is the exposure time (sec), and $E$ is the A/D conversion unit of the CCD (3.74 electron/count), respectively. In this calibration, the sensitivity for each observation mode and for each channel was determined by using 
these parameters. The solid angle is different for each observation mode since the size of a single bin is different (see Section 2.3). The transmittance of the optics and the quantum efficiency of the CCD are different for each channel. In this calibration, image data with three different exposure times and three different emission intensities were recorded for each MAC channel, and the results are summarized in Table 4.

\section{Operation}

The Reimei operation uses a 3-m antenna located at the JAXA/ISAS Sagamihara campus for a command uplink. The uplink speed is $1 \mathrm{kbps}$, and the downlink speed is up to $131 \mathrm{kbps}$ with an S-band system. In order to observe auroral phenomena in the polar region, it is necessary to transmit "Stored Command (SC)" when Reimei is visible from the JAXA/ISAS Sagamihara campus. SC includes, not only observation commands, but also commands for attitude control of the satellite, because the pre-determined attitude of the satellite has to be taken for observation. Though SC makes it possible to carry out the successive observation of auroras, SC has to be uplinked at least every 2 or 3 days due to the limited size of SC, 3864 bytes. Prior to sending SC, the operation is checked using a ground-based simulator (as will be described in detail in the following), to confirm that the sequence does not force the satellite into fatal conditions, e.g., the angle between the normal to the solar panel and the sun exceeds the limit, 25 degrees.

In order to achieve simultaneous observations of image and aurora particle measurements, the field of the MAC must be pointed at the footprint of the magnetic field line threading the satellite. In addition, the vectors perpendicular to the field of view of the ESA/ISA, which is $4 \times 300$ degrees, must be perpendicular to the magnetic field line threading the satellite, so that these particle analyzers observe particle energies with a full pitch angle. A schematic drawing of this observation is shown in Fig. 5. In order to control the satellite attitude for this observation, a tool was developed that simulates satellite attitudes with computer code. Files of orbital predictions and the magnetic field data based on the IGRF model are used as input data for this tool. The pointing vector of the MAC can be computed from the magnetic footprint data at the $110 \mathrm{~km}$ altitude and the predicted satellite location. Furthermore, by referencing the direction of the magnetic field at the location of the satellite, a vector of the ESA/ISA field of view is also computed. The simulator computes a feasible satellite attitude for every minute. At the same time, the sun angle of the solar panel is also computed for every attitude. In addition, it checks whether the footprint locations are in the auroral oval and whether the satellite is sunlit. If the sun angle to the solar panel is within the limit, 25 degrees, and neither the magnetic footprint nor the satellite are sunlit, the start time of the observation and the three vectors for Reimei at that time are output. The 3 -axes vectors for the satellite are converted to a vector composed of 4 elements, called " $q$-values." A command sequence for attitude control is generated from these " $q$-values" by another tool, which was developed by the engineering team for Reimei. Using these computed attitude values, as described above, a lot of

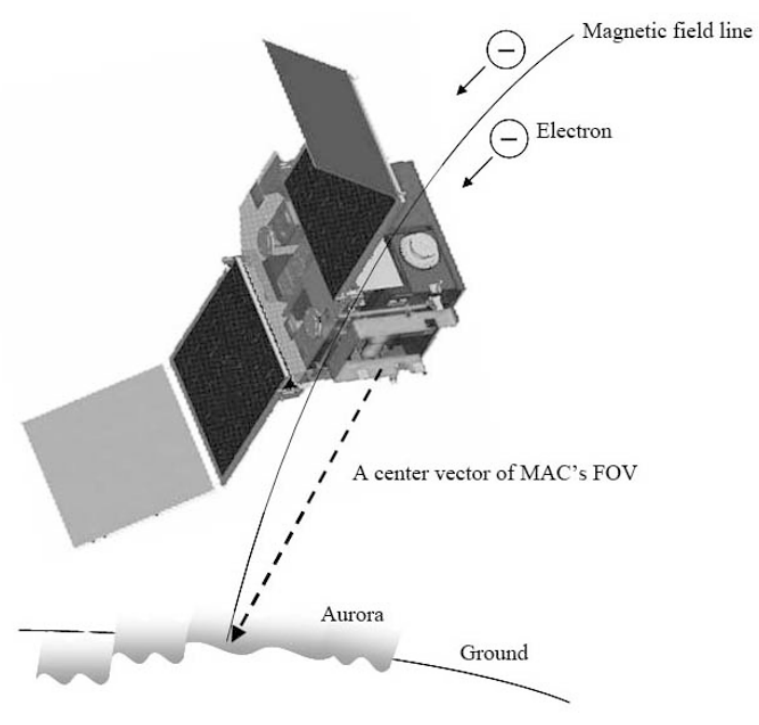

Fig. 5. Schematic drawing of the simultaneous observation of image and particle measurements. In this observation, the field of view of the MAC is pointed at the footprint of the magnetic field line threading the satellite.

simultaneous observation data have been successfully obtained, with a rate of more than $80 \%$ for all orbital passes. We also developed other tools for other observation modes. These are for pointing the MAC field of view to the locations of any associated ground-based instruments, or to the stars at any celestial coordinates. In these cases, from the orbital prediction and information about the ground-based instrument's location, the tool computes 3-axes vectors for the satellite for every minute. Another tool checks whether the satellite is within the attitude limit, which is specified by the sun angle of the solar panel and the field of a star tracker (STT). The STT must see only the sky all the time, and should not be aimed at the ground, in order to determine a satellite's attitude. When other instruments (not only ground-based, but also other satellite-born) request an associated Reimei observation, an observation plan can be flexibly produced by using these tools. Until now, we have succeeded in various collaborative observations with ALIS, EISCAT/ESR radar, SuperDARN radar, the all sky camera in Syowa, and THEMIS ground-based observatories, including Athabasca, Formosat-2, and AKEBONO satellite.

\section{Results}

By using the method for generating an operational plan described in the previous section, we have been carrying out observations with the MAC continuously since the launch of Reimei. The first results of auroral imaging observations are presented as follows.

An example of successive aurora image data obtained by MAC with "Mode-0" is shown in Fig. 6. This event was observed at around 09:31 UT on December 22, 2005 in the northern hemisphere. Although the exposure of each channel was repeated every $120 \mathrm{msec}$, images in Fig. 6 are presented at an interval of about $1 \mathrm{sec}$ due to space limitations. The upper, middle, and bottom rows indicate auroral images 


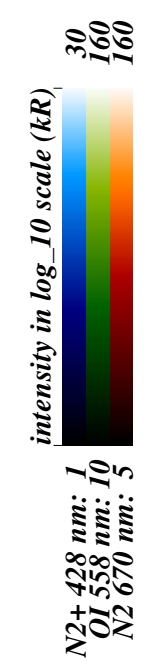

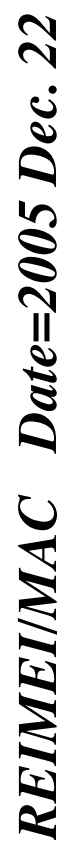
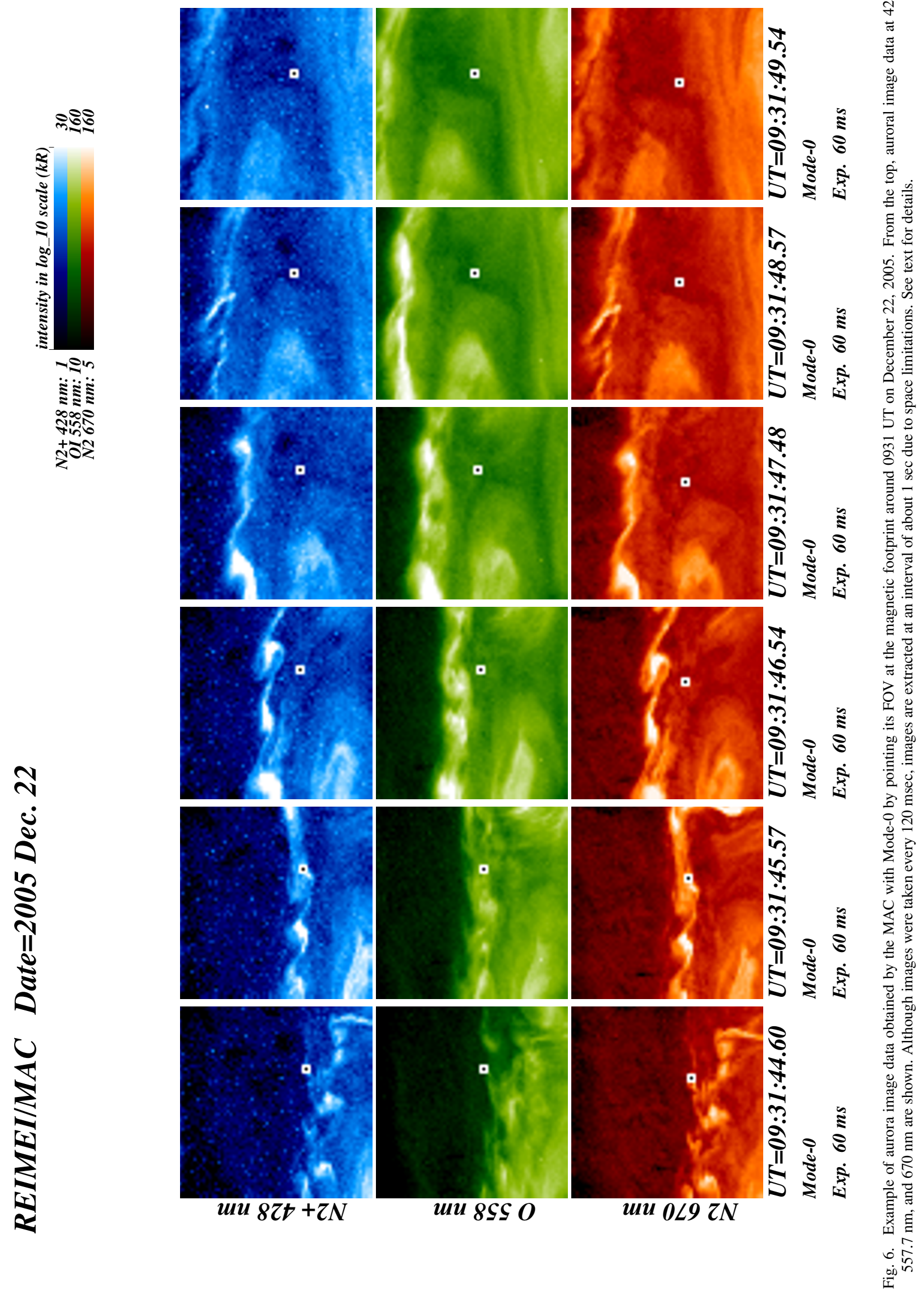

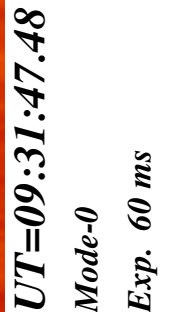

홍

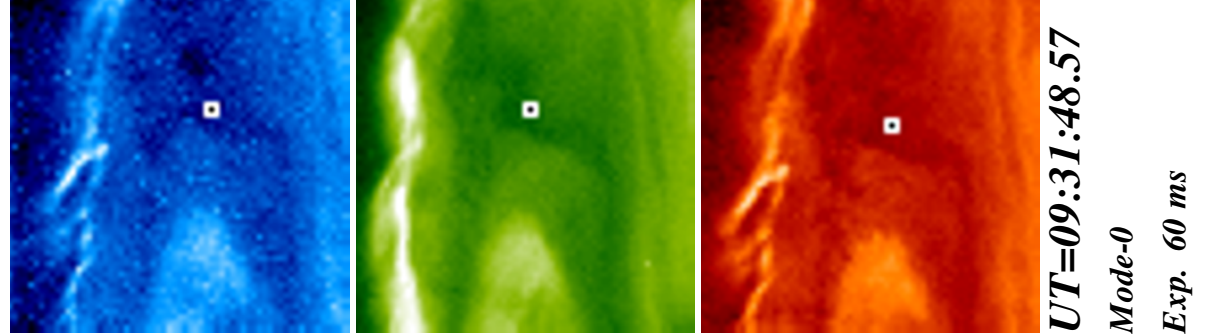

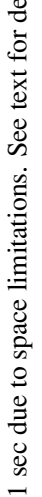




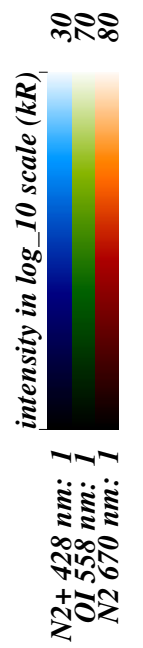

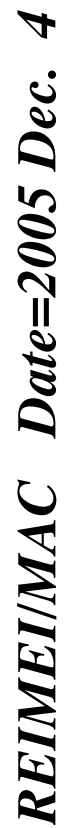

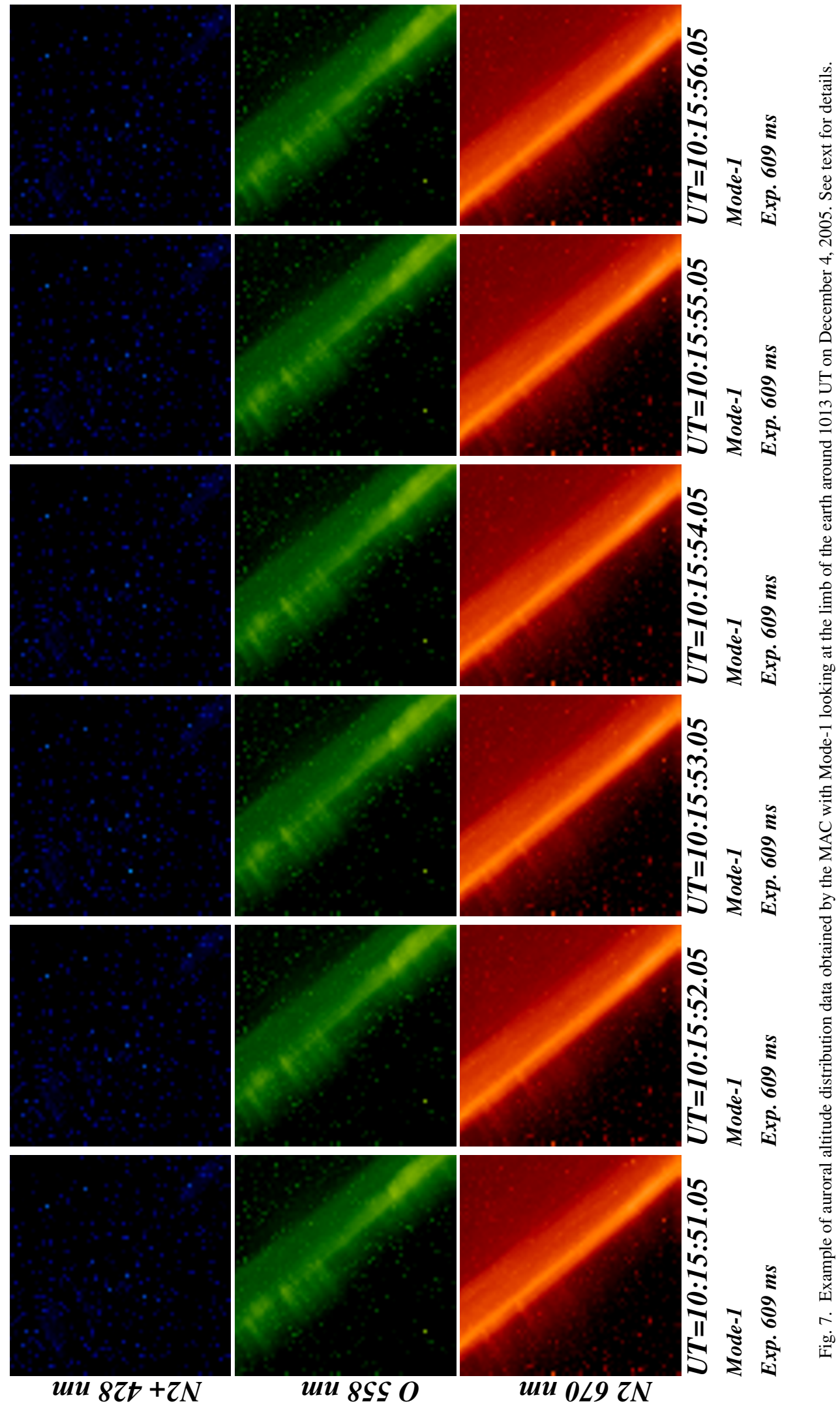


at wavelengths of $427.8 \mathrm{~nm}, 557.7 \mathrm{~nm}$, and $670 \mathrm{~nm}$, respectively. In all the images, geographic north is approximately upward, geographic east is to the right, and the area mapped at the $100 \mathrm{~km}$ altitude is $\sim 70 \times 70 \mathrm{~km}$. A white square denotes the location of the magnetic footprint estimated from the IGRF geomagnetic model. The continuous motion of the aurora seen from the bottom to the top is mainly due to the orbital motion of the satellite.

In this particular event, active discrete auroras with vortex street and shear flows are clearly seen in the poleward edge of the bright auroral region throughout this period. These shear flows containing vortices show fine structures and complicated motions, approximately along the eastwest direction. The auroral intensity was about 160 kiloRayleighs (KR) for $557.7 \mathrm{~nm}$ emission, and the size of vortex was approximately $3-10 \mathrm{~km}$. In the lower latitude region of these vortices, a large vortex/curl structure with a size of about $30 \mathrm{~km}$ appears from the west. Its shape is seen more clearly in the $427.8 \mathrm{~nm}$ emission and $670 \mathrm{~nm}$ emission than in the $557.7 \mathrm{~nm}$ emission, because the lifetime of the $557.7 \mathrm{~nm}$ emission is much longer than that of the other two emissions. The energy spectra of the precipitating electrons simultaneously observed by the ESA showed intense inverted-V structures with a peak energy of more than $12 \mathrm{keV}$, indicating the existence of quasi-electrostatic fieldaligned accelerations. In addition, supra-thermal energydispersed electron precipitations occurred in the inverted$\mathrm{V}$ structure, suggesting that the inertial Alfven waves also contribute to the electron acceleration, though the spectra are not shown in this paper. As shown here, it is evident that the MAC has a sufficient performance for achieving our scientific purpose of clarify the fine-scale dynamics of a bright aurora.

An example of the auroral altitude distribution obtained by the MAC with "Mode-1" is shown in Fig. 7. This event was observed at 10:13 UT on December 4, 2005. The format of the time sequence and wavelength was the same as in Fig. 6. The limb of the earth is seen from the top-left to the bottom-right, and the upward direction from the surface of the earth is bottom-leftward. The auroral emissions are seen in the $557.7 \mathrm{~nm}$ and $670 \mathrm{~nm}$ data above the surface of the earth throughout this period, while there are few $427.8 \mathrm{~nm}$ emissions. Oxygen and $\mathrm{OH}$ airglow emissions are also clearly seen in $557.7 \mathrm{~nm}$ and $670 \mathrm{~nm}$ data, respectively, suggesting that the MAC data are also useful for studying airglow. Accordingly, it is concluded that we have also succeeded in obtaining successive images of the altitude distribution of the aurora and airglows.

\section{Summary}

In order to investigate the auroral fine structure, the Reimei satellite was launched into polar orbit at an altitude of $\sim 630 \mathrm{~km}$ on August 23, 2005. We developed the MAC instrument for obtaining auroral image data at three wavelengths simultaneously. Since the electronics of the MAC, including the CCD, consisted of off-the-shelf commercial products, we qualified the performance for the space environment as summarized in the following. First, CCD noise could be reduced sufficiently to observe weak auroral emissions by using thermal paths made of graphite sheets. Secondly, in the radiation test, it was shown that the MAC is durable against radiation during the nominal observation period of Reimei. Thirdly, we developed a highperformance optical system to obtain precise auroral images, and succeeded in putting the CCD surface at a focal plane with an accuracy better than $10 \mu \mathrm{m}$. Lastly, in vibration and shock tests, it was demonstrated that the MAC was durable and that any misalignment of the CCD from the launch conditions was negligible. Furthermore, we developed software for generating operational plans by computing a sequence of attitude control for the simultaneous observation of image and particle measurements. After the launch, it was confirmed that the scientific instruments on board Reimei showed sufficient performance as expected and that the attitude could be correctly controlled. So far, lots of interesting events involving the fine-scale dynamics of auroras have been obtained with the MAC and ESA/ISA simultaneously. The Reimei data are available through the Internet.

Acknowledgments. We thank all members of the Reimei project team for their extensive effort toward the success of the Reimei mission. We thank Mr. Yukio Kaneko for manufacturing the body of the MAC. We thank Hamamatsu Photonics K.K. for manufacturing the CCD system of the MAC.

\section{References}

Asamura, K., D. Tsujita, H. Tanaka, Y. Saito, T. Mukai, and M. Hirahara, Auroral particle instrument onboard the INDEX satellite, Adv. Space Res., 32, 375-378, 2003.

Borovsky, J. E., Auroral Arc Thicknesses as Predicted by Various Theories, J. Geophys. Res., 98, 6101-6138, 1993.

Carlson, C. W., R. F. Praff, and J. G. Watzin, The fast auroral snapshot (FAST) mission, Geophys. Res. Lett., 25(12), 2013-2016, 1998.

Cattell, C., S3-3 Satellite instrumentation and data, in The IMS Source Book, edited by C. Russell and D. Southwood, p. 91, AGU, Washington, D.C., 1982.

Frey, H. U., G. Haerendel, J. H. Clemmons, M. H. Bochm, J. Vogt, O. H. Bauer, D. D. Wallis, L. Blomberg, and H. Lühr, Freja and ground-based analysis of inverted-V events, J. Geophys. Res., 103, 4303-4314, 1998.

Hoffman, R. A. and E. R. Schmerling, Dynamic Explorer program: An overview, Space Sci. Instrum., 5, 345-348, 1981.

Lundin, R., G. Haerendel, and S. Grahn, The Freja project, Geophys. Res. Lett., 21(17), 1823, 1994.

Maggs, J. E. and T. N. Davis, Measurements of the thicknesses of auroral structures, Planet. Space Sci., 16, 205, 1968.

McFadden, J. P., C. W. Carlson, and R. E. Ergun, Microstructure of the auroral acceleration region as observed by FAST, J. Geophys. Res., 104, 14453-14480, 1999.

Oya, H. and K. Tsuruda, Introduction to the Akebono (EXOS-D) satellite observations, J. Geomag. Geoelectr., 42, 367-370, 1990.

Saito, H., T. Mizuno, K. Tanaka, Y. Sone, S. Fukuda, S. Sakai, N. Okuizumi, M. Mita, Y. Fukushima, M. Hirahara, K. Asamura, T. Sakanoi, A. Miura, T. Ikenaga, and Y. Masumoto, AN-OVERVIEW AND INITIAL IN-ORBIT STATUS OF "INDEX" SATELLITE, 56th International Astronautical Confernce, IAC-05-B5.6.B.05, 2005.

Sakanoi, T., H. Fukunishi, and T. Mukai, Relationship between fieldaligned currents and inverted-V parallel potential drops observed at midaltitudes, J. Geophys. Res., 100, 19343-19360, 1995.

Sakanoi, T., S. Okano, Y. Obuchi, T. Kobayashi, M. Ejiri, K. Asamura, and M. Hirahara, Development of the multi-spectral auroral camera onboard the INDEX satellite, Adv. Space Res., 32, 379-384, 2003.

Schriver, D., M. Ashour-Abdalla, R. J. Strangeway, R. L. Richard, C. Klezting, Y. Dotan, and J. Wygant, FAST/Polar conjunction study of field-aligned auroral acceleration and corresponding magnetotail drivers, J. Geophys. Res., 108, COA21-1, 2003.

Y. Obuchi (e-mail: obuchi@genesia.co.jp), T. Sakanoi, A. Yamazaki, T. Ino, S. Okano, Y. Kasaba, M. Hirahara, Y. Kanai, and N. Takeyama 